\title{
Stable Robust Adaptive Control of Induction Motors with Unknown Parameters
}

\author{
Ibrahim Fahad Jasim \\ Electrical and Electronic Engineering Department \\ College of Engineering, University of Kerbala \\ Kerbala, Iraq \\ E-mail: ibrahim.jasim@ieee.org
}

\begin{abstract}
This paper presents a new strategy for controlling induction motors with unknown parameters. Using a simple linearized model of induction motors, we design robust adaptive controllers and unknown parameters update laws. The control design and parameters estimators are proved to have global stable performance against sudden load variations. All closed loop signals are guaranteed to be bounded. Simulations are performed to show the efficacy of the suggested scheme.
\end{abstract}

Keywords- Adaptive Robust Control; Induction Motors; Nonlinear Systems

\section{INTRODUCTION}

Induction motors are very popular in industrial and domestic applications. So, controlling this type of machines is a very important aspect to both control and power practitioners. Early control schemes implementing decoupled control of machine flux and torque are exemplified by FieldOriented Control (FOC) as proposed by Blaschke [1] and Leonhard [2]. The main idea of the FOC is to resolve the stator current into two parts; one controls the torque production and the other controls the flux. Thus the torque and flux would be controlled independently. However complete decoupling was not yet achieved. In [3-5], feedback linearization was successfully used to obtain a complete decoupling of torque and flux control, opening up a new direction of controlling the induction motors that torque (with position and velocity) and flux can be controlled separately.

The advent of control theory caused a significant impact on the induction motors control. Both adaptive control and sliding mode concept was used to control the induction motors with known parameters and promising results were obtained (see [6] and references therein). Moreover, adaptive fuzzy sliding mode control was successfully used to have a desirable position tracking error for induction motors [7] with unknown parameters. However, the technique described in [7] has the following drawbacks:

1. The fuzzy approximator fitted to operate within a restricted region of operation.

2. Only the subsystem corresponds to the torque is controlled. The flux remains uncontrolled.

In this paper, we address the problem of controlling the induction motor with unknown parameters. Both decoupled subsystems of the induction motor are taken into account. The angular position, speed, and flux are controlled to follow a prescribed reference signals.

The rest of the paper is organized as follows. In section 2, we present the control problem of the induction motor to be considered throughout this paper. The robust adaptive controllers and unknown parameters estimators are given in section3. Section 4 illustrates the simulation results and section 5 summarizes the concluding remarks.

\section{PROBLEM STATEMENT}

The mathematical model of a field oriented current command induction motor can be written as $[4,8]$ :

$$
\begin{aligned}
& \frac{d \theta}{d t}= \\
& \frac{d \omega}{d t}=\mu \psi_{d} l_{q r}-\frac{f}{I} \omega- \\
& \frac{d \psi_{d}}{d t}=-\eta \psi_{d}+\eta M \\
& \frac{d \rho}{d t}=n_{p} \omega+\frac{\eta_{i}}{i}
\end{aligned}
$$

With

$$
\eta \triangleq
$$

Where is the shaft angular displacement, is the angular speed, is the combined shaft and load inertia, is the number of pole pair, and are the rotor resistance and inductance respectively, is the motor mutual inductance, is the flux magnitude, is the flux angle, is the quadrature current component, and is the direct axis current component.

Normally, is used to estimated the current and (or) speed, and since it is an angle, then its value is always limited between and 3i. So, it is always bounded and it rarely to be a control objective.

The parameters $\mathrm{f}, \mathbb{J}, \mathfrak{\eta}$, and $\mathrm{M}$ are assumed to be unknown. All states are assumed to be available for measurement.

Now, let: 
$\mathrm{u}_{1}=\mathbb{1}_{\mathrm{qr}} \mathrm{uW}_{\mathrm{d},} \mathrm{u}_{2}=\mathrm{i}_{\mathrm{dr}}, \mathrm{a}_{1}=\frac{\mathrm{f}}{\mathrm{I}}, \mathrm{a}_{2}=\frac{1}{\mathbb{1}}, \mathrm{a}_{\mathrm{a}}=\mathrm{n}, \mathrm{a}_{4}=\mathrm{\eta} \mathrm{M}$

Then the model given in (1) can be rewritten as:

$\frac{d \theta}{d t}=\omega$

$\frac{d \omega}{d t}=-a_{1} \omega-a_{2} T_{L}+\mathbb{M}_{1}$

$\frac{d \Psi_{a}}{d t}=-a_{g} \Psi_{d}+a_{4} u_{2}$

The objective of the paper is to derive robust adaptive control laws (for $u_{1}$ and $u_{2}$ ) and parameters update laws for $a_{1}, a_{2}, a_{\mathbb{a}}$, and $a_{4}$ such that $\theta_{x} \omega_{x}$ and $\Psi_{\mathbb{d}} \rightarrow \theta_{x g f}, \omega_{\text {yef }}$, and $\Psi_{\text {dref }}$ respectively as $t \rightarrow \infty$ provided that all closed loop signals are bounded. However, the following assumptions are needed to be satisfied:

A1. All states $\theta_{s} \omega_{x}$ and $\Psi_{d}$ are assumed to be available for measurement.

A2. All reference signals $\theta_{\text {ref }}, w_{\text {ref }}$, and $\psi_{\text {dref }}$ are assumed to be bounded.

A3. The parameters $a_{1}, a_{2}, a_{2}$ and $a_{4}$ are bounded.

\section{ROBUST ADAPTIVE CONTROL DESIGN}

Before we present the main theorem of this paper, we define several concepts. Let:

$$
\begin{aligned}
& e_{1}=\theta-\theta_{\text {ref }} \\
& e_{2}=\omega-\omega_{\text {ref }} \\
& e_{a}=\Psi_{d}-\Psi_{d r e f}
\end{aligned}
$$

It is clear that the dynamic model given in (2) consists of two decoupled subsystems, say (2.a, 2.b) and (2.c). So, we shall define two sliding surfaces, say $s_{1}$ and $s_{2}$, as shown below:

$$
\begin{array}{ll}
s_{1}=\left(\frac{d}{d t}+\lambda_{1}\right) e_{1} & \lambda_{1}>0 \\
s_{2}=\left(\frac{d}{d t}+\lambda_{2}\right)^{0} e_{a} & \lambda_{2}>0 \\
\therefore s_{2}=e_{3} &
\end{array}
$$

Taking the time derivative for (4.a) and (4.b), we obtain:

$s_{1}^{\infty}=e_{1}^{m}+\lambda_{1} e^{n}$ $\therefore s_{1}^{x}=\theta-\theta_{\text {ref }}+\lambda_{1}\left(\theta-\theta_{\text {ref }}\right)$

$s_{2}^{n}=e_{n}^{n}$

${ }_{m} s_{2}^{n}=\mathbb{H}_{d}-\mathbb{H}_{\text {dref }}$

Note: It was shown that the filtered errors given in (4.a) and (4.b) has the following properties: (i) the equations $s_{1}(t)=0$ and $s_{2}(t)=0$ define time-varying hyperplanes in $R^{2}$ and $R$, on which the tracking errors $e_{1}, e_{2}$, and $e_{a}$ decays ecponetially to zero. (ii) if $e(0)=0$ and $\|s(t)\| \leq \varepsilon$ with constant $\varepsilon$, then

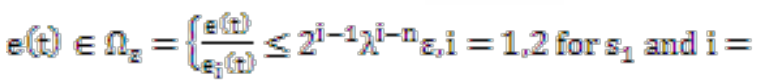
1 for $s_{2}$ \}

for all $t \geq 0$. (iii) if $e(0) \neq 0$ and $\|s(t)\| \leq \varepsilon$, then $e(t)$ will converge to $\Omega_{z}$ within a time constant $\frac{[\mathrm{m}-1])}{\mathrm{d}}$ (see $\left.[9,10]\right)$.

Define the parameters errors to be:

$\mathrm{a}_{1}=\mathrm{a}_{1}-\mathrm{a}_{1}^{*}$

$\tilde{a}_{2}=\hat{a}_{2}-a_{2}^{*}$

$\mathrm{a}_{\mathrm{a}}=\mathrm{a}_{\mathrm{a}}-\mathrm{a}_{\mathrm{a}}$

$\mathrm{a}_{4}=\mathrm{a}_{4}-\mathrm{a}_{4}$

Define also the modified filtered error:

$s_{z 1}=s_{1}-\varepsilon_{1} \operatorname{sat}\left(\frac{s_{1}}{s_{1}}\right)$

$s_{z 2}=s_{2}-\varepsilon_{2} \operatorname{sat}\left(\frac{s_{2}}{s_{2}}\right)$

Theorem: For the induction motor given in (2) satisfying A1, $\mathbf{A 2}$ and A3, the controllers given in (8.a and 8.b) along with the parameters update laws (8.c, 8.d, 8.e, and 8.f) can guarantee global system stability and enhanced tracking performance.

$\mathrm{u}_{1}=-\mathrm{k}_{\mathrm{d} 1} s_{1}+\hat{\mathrm{a}}_{1} \omega+\hat{\mathrm{a}}_{2} \mathrm{~T}_{\mathbb{L}}-\lambda_{1}\left(\omega-\omega_{\mathrm{ref}}\right)$

$\mathrm{u}_{2}=\frac{1}{\hat{\mathrm{A}}_{4}}\left(-\mathrm{k}_{\mathrm{d} 2} \mathrm{~s}_{2}+\hat{\mathrm{a}}_{\mathrm{g}} \Psi_{\mathrm{d}}+\Psi_{\mathrm{dref}}\right)$

$\mathrm{a}_{1}=-\mathrm{\gamma}_{1} \cos \mathrm{z}_{\mathrm{z}}$

$\hat{a}_{2}=-\gamma_{2} \hat{a} s_{21}$

$\mathrm{a}_{\mathrm{a}}=-\mathrm{V}_{\mathrm{a}} \Psi_{\mathrm{d}} \mathrm{s}_{\mathrm{z} 2}$ 


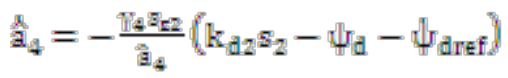

Proof: Consider the Lyapunov candidate:

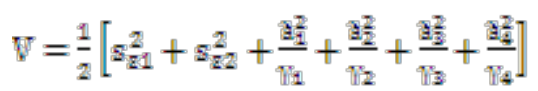

Taking the time derivative of (9), we obtain:

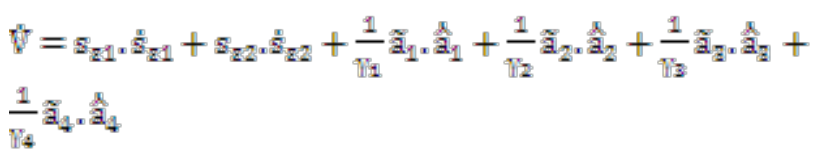

From (7.a and 7.b), we can easily conclude that:

$\mathrm{s}_{\mathrm{g} 1}=\mathrm{s}_{1}$

$s_{z 2}=s_{2}$

Using (5.a and 5.b), then (10) can be rewritten as:

$$
\begin{aligned}
& v=s_{z 1} \cdot\left(\theta-\theta_{\text {ref }}+\lambda_{1}\left(\theta-\theta_{\text {ref }}\right)\right)+s_{z z} \cdot\left(\psi_{a}-\psi_{a r e f}\right) \\
& +\frac{1}{\mathbb{T}_{1}} \widetilde{a}_{1} \cdot \hat{a}_{1}+\frac{1}{\mathbb{T}_{2}} \widetilde{a}_{2} \cdot \hat{a}_{2}+\frac{1}{\mathbb{T}_{3}} \widetilde{a}_{1} \cdot \hat{a}_{11}+\frac{1}{\mathbb{T}_{4}} \widetilde{a}_{4} \cdot \hat{a}_{4}
\end{aligned}
$$

Substituting (2.a, 2.b, and 2.c) into (11), then we obtain:

$$
\begin{aligned}
& V=s_{\mathbb{R} 1} \cdot\left(-a_{1} \omega-a_{2} T_{L}+\mathbb{u}_{1}-\theta_{\mathrm{ref}}+\lambda_{1}\left(\omega-\omega_{\mathrm{ref}}\right)\right) \\
& +s_{z 2} \cdot\left(-a_{n} \Psi_{a}+a_{4} u_{2}-\Psi_{d r e f}\right)+\frac{1}{\gamma_{1}} \widetilde{a}_{1} \cdot a_{1}+\frac{1}{\gamma_{2}} \widetilde{a}_{2} \cdot \hat{a}_{2} \\
& +\frac{1}{\mathbb{W} 3} a_{1} \cdot a_{1}+\frac{1}{\mathbb{T}_{4}} a_{4} \cdot a_{4}
\end{aligned}
$$

Using the controllers defined in (8.a and 8.b), then we obtain:

$$
\begin{aligned}
& V=s_{21} \cdot\left(-a_{1} \omega-a_{2} T_{\mathbb{L}}+\left(-k_{d 1} s_{1}+\hat{a}_{1} \omega+\hat{a}_{2} T_{\mathbb{L}}\right.\right. \\
& \left.\left.-\lambda_{1}\left(\omega-\omega_{\text {ref }}\right)\right)-\theta_{\text {ref }}+\lambda_{1}\left(\omega-\omega_{\text {ref }}\right)\right)+s_{z 2} \cdot\left(-a_{a} \Psi_{a}+\right. \\
& \left.a_{4}\left(\frac{1}{a_{4}}\left(-k_{d 2} s_{2}+\hat{a}_{a} \Psi_{d}+\Psi_{d r e f}\right)\right)-\Psi_{d r e f}\right)+ \\
& \frac{1}{\mathbb{W}_{1}} \widetilde{a}_{1} \cdot \hat{a}_{1}+\frac{1}{\mathbb{T}_{2}} \widetilde{a}_{2} \cdot \hat{a}_{2}+\frac{1}{\mathbb{W}_{3}} \widetilde{a}_{1} \cdot \hat{a}_{1}+\frac{1}{\mathbb{T}_{4}} \widetilde{a}_{4} \cdot \hat{a}_{4}
\end{aligned}
$$

After several simple mathematical manipulations for (13), we can obtain:

$V=-k_{\mathrm{d} 1} \cdot s_{\mathrm{z} 1} \cdot s_{1}-k_{\mathrm{d} 2} \times s_{\mathrm{z} 2} \cdot s_{2}+\widetilde{a}_{1}\left(s_{\mathrm{z} 1} \cdot \omega+\frac{1}{\gamma_{1}} \widetilde{\mathrm{a}}_{1}\right)$

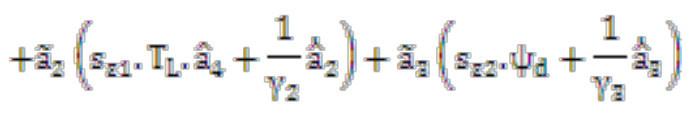

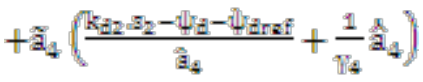

Using the parameters updates laws given in (8.c, 8.d, 8.e, and 8.f) and the relation given in (7.a and 7.b), we obtain:

$$
\begin{aligned}
& V=-k_{\mathrm{d} 1} \times s_{z 1} \times\left(s_{z 1}+\varepsilon_{1} \times s a t\left(\frac{s_{1}}{\varepsilon_{1}}\right)\right)-k_{d 2} \times s_{z 2} \cdot\left(s_{z 2}\right. \\
& \left.+\varepsilon_{2} \times \operatorname{sat}\left(\frac{s_{2}}{s_{2}}\right)\right)
\end{aligned}
$$

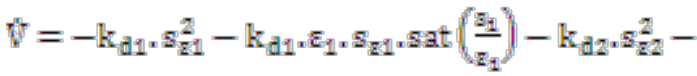

$$
\begin{aligned}
& \mathrm{k}_{\mathrm{d} 2} \times \varepsilon_{2} \times \mathrm{s}_{\mathrm{z2}} \times \mathrm{sat}\left(\frac{\mathrm{s}_{2}}{\mathrm{~s}_{2}}\right)
\end{aligned}
$$

Then can satisfy inequality below:

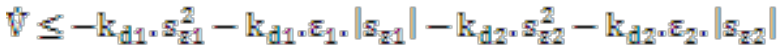

From (17), it is clear that $\Xi_{z 12} \in \mathcal{L}_{2} \cap \mathcal{L}_{\mathrm{z}}$ and $\widetilde{a}_{1}, \tilde{a}_{2}, \widetilde{a}_{\mathbb{2}}, \widetilde{a}_{4} \in \mathcal{L}_{n}$. Since $a_{1}, a_{2}, a_{\mathbb{g}}$ and $a_{4}$ are bounded and

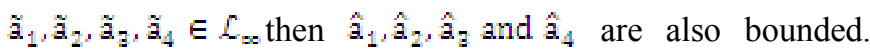
Using (5.a and 5.b), then we can easily conclude that

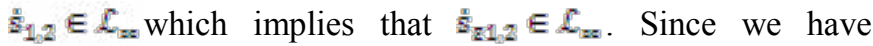
$s_{\mathrm{z} 1,2} \in \mathcal{L}_{2} \cap \mathcal{L}_{\mathrm{sz}}$ and $\quad \mathrm{s}_{\mathrm{z} 12} \in \mathcal{L}_{\mathrm{ms}}$, then $\mathrm{s}_{\mathrm{z} 1,2} \rightarrow 0$ as $t \rightarrow \infty$ according to Barbalat's lemma. This would force $\Xi_{s} \widetilde{\omega}_{s}$ and $\widetilde{\Psi}_{\mathbb{E}}$ for converging to $\Omega_{\mathrm{z} 12}$.

\section{Simulation Results}

Simulations were carried out for an induction machine with the following parameters:

Connection type is $\mathrm{Y}$, voltage rating is $380 \mathrm{~V}$, current rating is $5 \mathrm{~A}$, number of phase $3 \mathrm{ph}$, rated power is $2.2 \mathrm{~kW}$, frequency is $50 \mathrm{~Hz}$, rated speed is $1430 \mathrm{rpm}, R_{\gamma}$ is $1.7 \Omega, \mathbb{L}_{y}$ is $0.34 \mathrm{H}$, $R_{g}$ is $3.5 \Omega, \quad \mathbb{L}_{g}$ is $0.31 \mathbb{H}, \mathbb{L}_{\mathrm{sm}}$ is $0.29 \mathbb{H}, \boldsymbol{l}$ is $4.78 \times 10^{-1} \mathrm{Nm} / \mathrm{s}^{2}$, and $f$ is $5.34 \times 10^{-9} \mathrm{Nms} / \mathrm{rad}$.

The position, velocity and flux reference signals are:

$$
\begin{aligned}
& \theta_{\text {ref }}=\sin (0.4 \pi \mathrm{t}), \omega_{\text {ref }}=0.4 \pi \cos (0.4 \pi \mathrm{t}) \\
& \psi_{d v e f}=0.5 \sin (0.4 \pi \mathrm{t})
\end{aligned}
$$

The load torque is assumed to be varying in a square wave fashion of $0.2 \mathrm{~Hz}$ frequency and peaks of +1 and -1 . 
Figures 1, 2, and 3 show the position, velocity, and flux tracking performance. It is clear that excellent tracking performance was obtained for all three states, say $\theta, \omega$, and $\Psi_{d e}$. As illustrated through the section 3and the theorem therein, all states errors would converge asymptotically to bounded region, say $\Omega_{\mathrm{z} 1_{2} 2}$. The boundary of this region can be specified by the designer through choosing appropriate values for $\varepsilon_{1,2}$ and $\lambda_{12}$ which is done through trade-off. Smaller values of $\lambda_{1,2}$ would cause the regions $\Omega_{z_{1,2}}$ to be smaller, however the convergence time would be increased. Similarly, smaller values of $\varepsilon_{1,2}$ would cause smaller regions of $\Omega_{R_{12} 2}$, however chattering would be caused spurring high frequencies that may constitute a threat to the overall system stability $[9,10]$. In our design we used the values of $\varepsilon_{1}$ and $\varepsilon_{2}$ to be 0.01 . For $\lambda_{1}$ and $\lambda_{2}$, we took the values of 1 and 0.1 respectively.

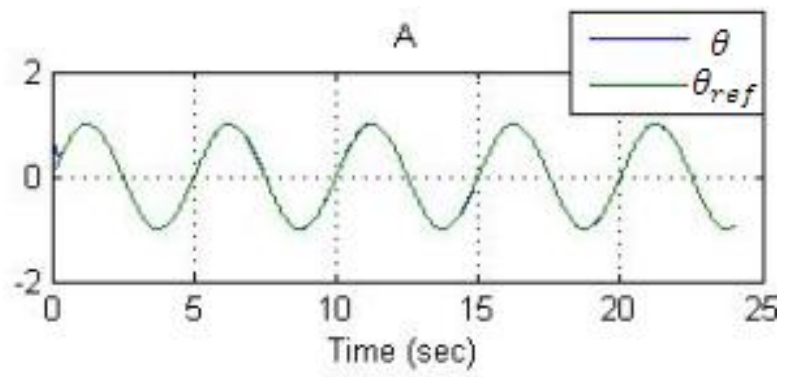

$\mathrm{B}$

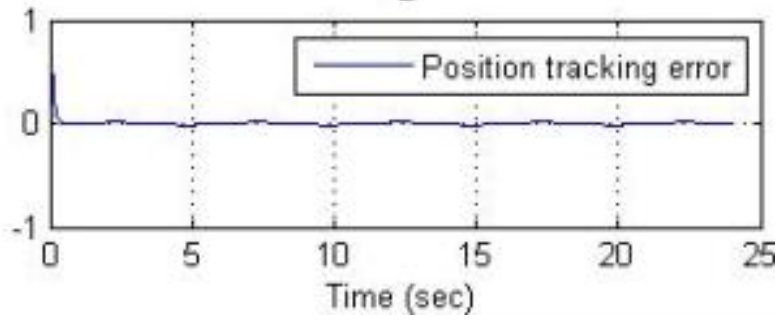

Figure 1 A. Reference and actual angular position (in rad) B. Position tracking error (in rad)
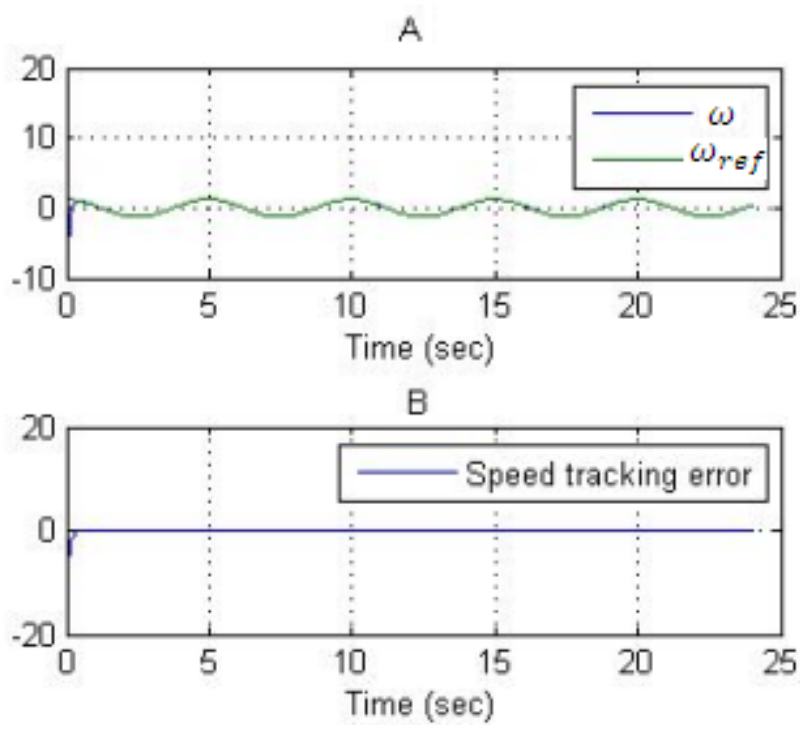

Figure 2 A. Reference and actual angular speed (in rad/sec) B. Speed tracking error (in rad/sec)

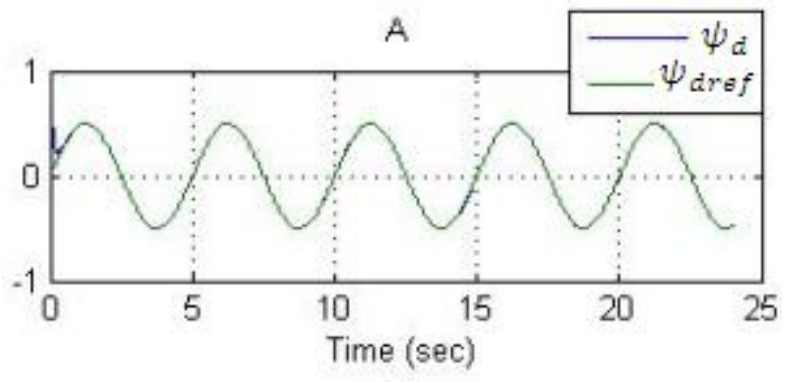

$\mathrm{B}$

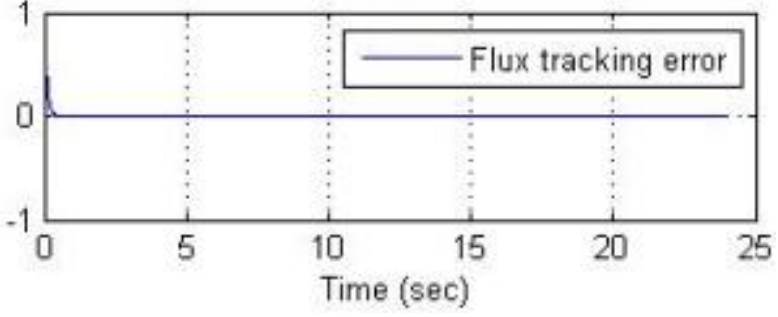

Figure 3 A. Reference and actual flux (in web) B. Flux tracking error (in web) 
A

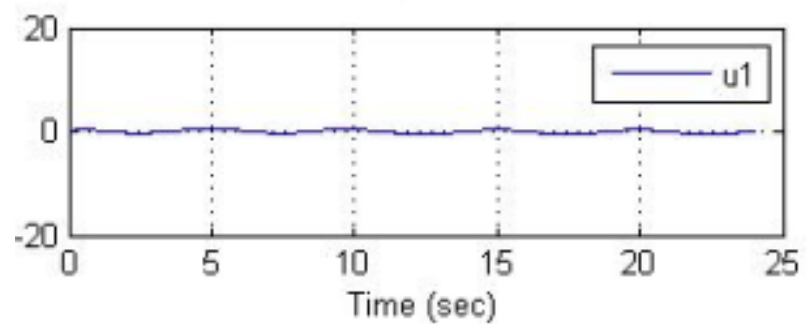

B

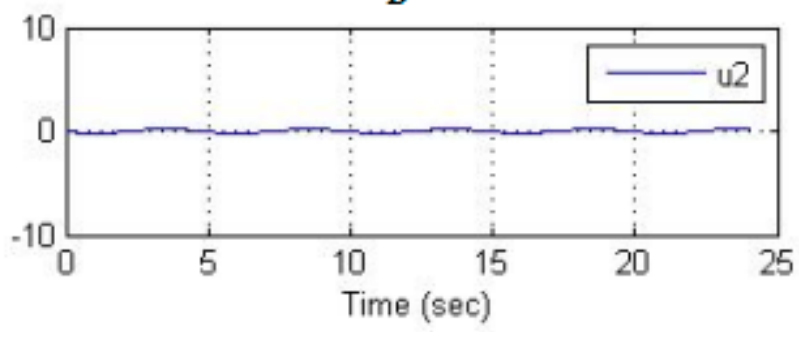

Figure 4 A. Torque control action $w_{1}$ B. Flux control action $u_{2}$

Both control actions are given in Figure 4. Both control actions are functions of the signals that were proved to be bounded. So, both of the control actions would be bounded (See Figure $3 \mathrm{~A}$ and $\mathrm{B}$ ). The parameters $\hat{\mathrm{Q}}_{1}, \hat{\mathrm{Q}}_{2}, \hat{\mathrm{Q}}_{\mathrm{Z}}$, and $\hat{\mathrm{Q}}_{4}$ ere also proved to be bounded and as per checking Figure 5, it is clear that all the parameters estimated are bounded as proved in the paper main theorem.

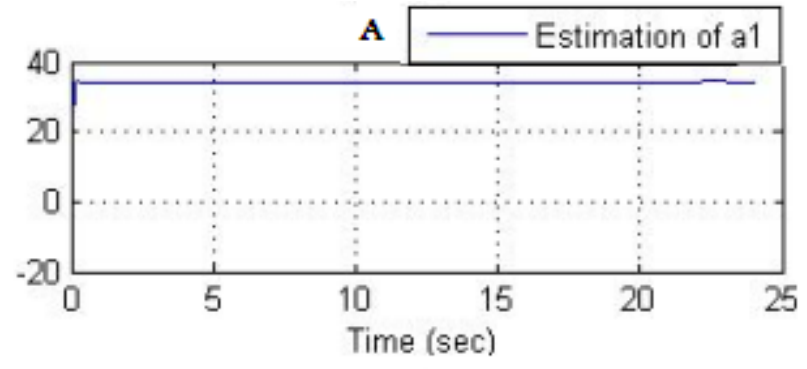

B
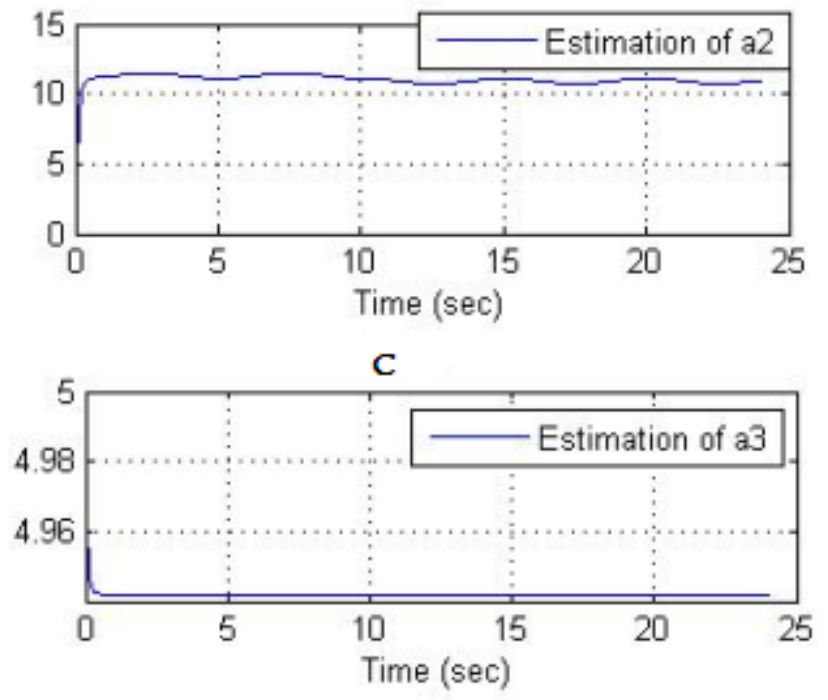

D

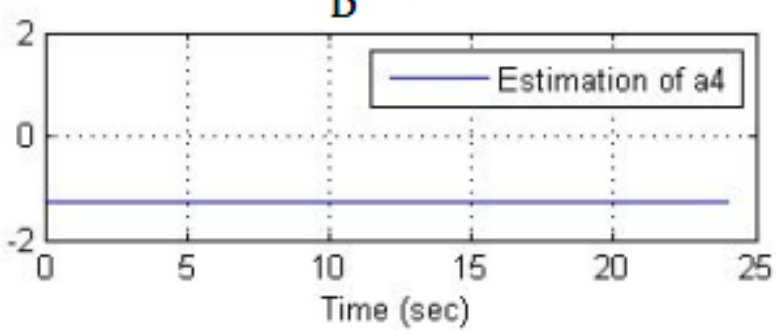

Figure 5 A. Estimation of $a_{1}$ B. Estimation of $a_{2}$ C. Estimation of $a_{2} D$. Estimation of 4

\section{CONCLUSION}

The control problem of induction motors with unknown parameters was addressed. Robust adaptive control laws were derived for both flux and torque dynamics. Estimators for the unknown parameters were also suggested. The suggested scheme was shown to have global stable performance with all closed loop signals guaranteed to be bounded. However, the control actions derived are of high initial values that may break the current constraints. So, future works should focus on deriving new control schemes that keeps the control actions to be within a prescribed bound in order to keep the currents constraint valid. 


\section{REFERENCES}

[1] F. Blaschke, " The principle of field orientation applied to transvector closed-loop control system for rotating field machines," Siemens Rev., vol. 34, no. 5, pp. 217-220, 1972.

[2] W. Leonhard, “ Microcomputer control of high dynamic performance ac- drives -A survey," Automatica, vol. 22, no. 1, pp. 1-19, 1986.

[3] D. I. Kim, and I. J. Ha, and M. S. Ko, " Control of induction motors via feedbak linearisation with input-output decoupling," Int. J. Control, vol. 51, no. 4, pp. 863-883, 1990.

[4] R. Marino, S. Peresada, and P. Valigi, "Adaptive input-output linearizing control of induction motors," IEEE Trans. Automat. Contr., vol.38, no.2, pp. 208-221, 1993.

[5] M. Bodson, J. Chiasson, and R. Novotnak, "High perforance induction control via input-output linearization,”, IEEE Contr. Syst. Magazine, vol. 4, no. 4, pp. 25-33, 1994.

[6] O. Barambones, A. J. Garrido, F. J. Maseda, and P. Alkorta, “ An adaptive sliding mode control law for induction motors using field oriented control theory," Proc. Of the 2006 IEEE Inter. Conf. on Cont. Appl., Munich Germany, Oct. 4-6, pp. 1008- 1013, 2006

[7] H. F. Ho, and K. W. E. Cheng, " Position control of induction motor using indirect adaptive fuzzy sliding mode control," $3{ }^{\text {rd }}$ Inter. Conf. on Power Elect. and Appl., pp., 2009

[8] J. Chiasson, " Modelling and high-performance control of electric machines,", IEEE Press Series on Power Engineering, John Wiley \& Sons, 2005.

[9] J. -J. E. Slotine, "Sliding controller design for nonlinear systems," I nt. J. Control, vol. 40, pp.435- 448, 1986.

[10] J. -J. E. Slotine, and W. Li, "Applied nonlinear control", Prentice- Hall Inc., 1991. 